\title{
GEOMETRY OF GENUS 8 NIKULIN SURFACES AND RATIONALITY OF THEIR MODULI
}

\author{
ALESSANDRO VERRA
}

\section{INTRODUCTION}

In this note we study Nikulin surfaces of genus 8 and their moduli. As typical at least in low genus, the family of surfaces to be investigated sits in a fascinating system of relations to other known geometric families. Our aim is to unveil one of these relations, namely that occurring between the moduli of Nikulin surfaces of genus 8 and the Hilbert scheme of rational sextic curves in the Grassmannian $G(1,4)$. We will work over an algebraically closed field $k$, char $k=0$.

A Nikulin surface of genus $g$ will be a K3 surface $S$ endowed with a pseudoample, primitive polarization $\mathcal{L}$ of genus $g$ and a line bundle $\mathcal{O}_{S}(M)$ such that $2 M \sim N$, where $N$ is the disjoint union of 8 copies of $\mathbf{P}^{1}$. In particular one has $g \geq 2$.

The corresponding moduli space of Nikulin surfaces of genus $g$ is known to be an equidimensional quasi projective variety of dimension 11. Its irreducible components are essentially characterized by the intersection index of $\mathcal{L}$ and $\mathcal{O}_{S}(2 M),[\mathrm{H}, \mathrm{GS}, \mathrm{SvG}$.

We will assume throughout all the paper that the intersection index of $\mathcal{L}$ and $\mathcal{O}_{S}(M)$ is zero.

The latter condition defines an integral component of the moduli space which exists for every $g$, differently from the other possible conditions. Moreover this is the unique irreducible component if $g \equiv 0 \bmod 4$. On the other hand the birational structure of it, when $g$ varies, is quite unknown. Recently its unirationality has been proven for $g \leq 7$, [FV, FV1, while no result on the Kodaira dimension seems to be known for $g \geq 9$. The main result proved in this paper is the following

THEOREM I The moduli space of genus 8 Nikulin surfaces is rational.

As a consequence, it is natural to ask wether the rationality of these moduli spaces could be true for lower genus $g \leq 7$. Let us briefly describe the method of proof and how it is related to a special family of rational normal sextic curves in the Plücker embedding of the Grassmannian $G(1,4)$.

Research supported by the PRIN Project 'Geometry of Algebraic Varieties' and by INdAM-GNSAGA. 
Assume that $S$ is a general Nikulin surface of genus 8 , then $\mathcal{H}:=\mathcal{L}(-M)$ is a very ample polarization of genus 6 . Moreover we have $\mathcal{H}(-M) \cong \mathcal{O}_{S}(A)$, where $A$ is a copy of $\mathbf{P}^{1}$ and $A$ has degree 6 with respect to $\mathcal{H}$.

The main idea behind the proof is to consider the Mukai bundle $\mathcal{E} \rightarrow S$ defined by the polarization $\mathcal{H}$. As is well known this is a stable rank 2 vector bundle with $h^{0}(\mathcal{E})=5$ and det $\mathcal{E} \cong \mathcal{H}$. Let $G(1,4) \subset \mathbf{P}^{9}$ be the Plücker embedding of the Grassmannian of lines of $\mathbf{P} H^{0}(\mathcal{E})^{*}$. It turns out that $\mathcal{H}$ defines an embedding $S \subset \mathbf{P}^{6}$, factoring through the classifying map $S \rightarrow G(1,4)$ defined by $\mathcal{E}$. Furthermore, it is also true that the restriction map $r: H^{0}\left(\mathcal{O}_{G(1,4)}(1)\right) \rightarrow H^{0}(\mathcal{H})$ is surjective. Let $\mathbf{P}^{6} \subset \mathbf{P}^{9}$ be the linear embedding induced by the dual of $r$, then we have

$$
A \subset S \subset G(1,4) \cap \mathbf{P}^{6} \subset \mathbf{P}^{9} .
$$

If $S$ is general $A$ is embedded as a rational normal sextic and the restriction $\mathcal{E}_{A}:=\mathcal{E} \otimes \mathcal{O}_{A}$ is the sheaf $\mathcal{O}_{\mathbf{P}^{1}}(3) \oplus \mathcal{O}_{\mathbf{P}^{1}}(3)$. We will consider the ruled surface $\mathbf{P} \mathcal{E}_{A}^{*}$ and its tautological embedding $R$ in $\mathbf{P}^{7}:=\mathbf{P} H^{0}\left(\mathcal{E}_{A}\right)^{*}$. Let $\mathbf{P}^{4}:=\mathbf{P} H^{0}(\mathcal{E})^{*}$ then the restriction $H^{0}(\mathcal{E}) \rightarrow H^{0}\left(\mathcal{E}_{A}\right)$ uniquely defines a linear projection

$$
p: R \rightarrow \mathbf{P}^{4} \text {. }
$$

For a Nikulin surface $S$ the map $p$ is not a generic linear projection of $R$. As we will see the projected surface $p(R)$ is a scroll with a curve of double points and this is actually a rational normal quartic curve $B$. More precisely $p(R)$ is the complete intersection of a quadric through $B$ and $S e c B$, the cubic hypersurface of the bisecant lines to $B$.

On the other hand this realization of $p(R)$ is used to show that $A$ admits a one dmensional family of bisecant lines which are contained in $G(1,4)$. Let $T$ be the intersection of the linear span of $A$ with $G(1,4)$ : we will show that $T$ is a smooth threefold. Let us say that $A \subset T$ is an embedding of special type. Along the paper we describe the special geometry of it and its relations to Nikulin surfaces. One can summarize all that as follows.

THEOREM II Let $A \subset T$ be a general embedding of special type and let $\mathcal{I}_{A}$ be its ideal sheaf. Then:

- A general member of $\left|\mathcal{I}_{A}(2)\right|$ is a Nikulin surface of genus 8 .

- For a general Nikulin surface $S$ of genus 8 there exists a special embedding $A \subset T$ such that

$$
S \in\left|\mathcal{I}_{A}(2)\right|
$$

This is the starting point for a quick conclusion of the proof of theorem I, saying that the moduli space of genus 8 Nikulin surfaces is rational.

We consider this note as a step in the study of moduli of Nikulin surfaces of low genus by similar geometric methods. As is well known a general member of the Hilbert scheme of $S$ in $G(1,4)$ is a general K3 surface of genus 6. Furthermore, by Mukai linear section theorem, a general K3 surface 
of genus $7 \leq g \leq 10$ can be constructed as a linear section of a suitable homogeneous space $\mathbb{S}_{g}$ and such a realization is unique up to Aut $\mathbb{S}_{g}$.

Describing Mukai realizations of Nikulin surfaces of genus $g=9,10,11$ appears to be, as in the case $g=8$, very rich of geometric connections and interesting for the study of the corresponding moduli spaces.

More in general the moduli space of Nikulin surfaces of genus $g$ deserves to be studied. In particular it could be tempting to compare the family of the moduli spaces of Nikulin surfaces of genus $g$ and the family of the moduli spaces of Enriques surfaces endowed with a genus $g$ polarization, since the two classes of surfaces admit some analogies.

For instance, in the case of Enriques surfaces, a recent theorem of Hulek and Gritsenko, $\mathrm{GH}$, shows that the irreducible components of the moduli spaces, when $g$ varies, distribute in finitely many birational classes: what happens in the case of Nikulin surfaces? Hopefully further work will be developed about the previous questions in the future.

\section{Notations AND PRELIMINARY RESUlts}

A $K 3$ surface of genus $g$ is a pair $(S, \mathcal{L})$ such that $S$ is a K3 surface and $\mathcal{L}:=\mathcal{O}_{S}(C)$ is a pseudoample, primitive element of Pic $S$ such that $C^{2}=2 g-2$. The moduli space of K3 surfaces of genus $g$ is an irreducible, quasi-projective variety of dimension 19 . It will be denoted as usual by $\mathcal{F}_{g}$.

As already outlined in the introduction a Nikulin surface of genus $g$ will be a triple $(S, \mathcal{L}, \mathcal{M})$ such that:

(1) $(S, \mathcal{L})$ is a K3 surface of genus $g$.

(2) $\mathcal{M}:=\mathcal{O}_{S}(M)$, where $2 M \sim N_{1}+\cdots+N_{8}$ and $N_{1} \ldots N_{8}$ are disjoint copies of $\mathbf{P}^{1}$.

(3) $\mathcal{L}$ and $\mathcal{M}$ are orthogonal in Pic $S$.

Note that $N_{i} N_{j}=-2 \delta_{i j}$ and that $M^{2}=-4$. We fix the notation

$$
N:=N_{1}+\cdots+N_{8} \text {. }
$$

Lemma 2.1. $h^{i}\left(\mathcal{O}_{S}(M)\right)=0$ for $i=0,1,2$.

Proof. Since $N$ is isolated and $2 M \sim N$, we have $h^{0}\left(\mathcal{O}_{S}(M)\right) \leq 1$. Let $h^{0}\left(\mathcal{O}_{S}(M)\right)=1$, then $M \sim E$ for an effective divisor $E$ and $2 E=N$. Since $N$ is reduced this is impossible, hence $h^{0}\left(\mathcal{O}_{S}(M)\right)=0$. Moreover we have $h^{0}\left(\mathcal{O}_{S}(-2 M)\right)=h^{0}\left(\mathcal{O}_{S}(-N)\right)=0$ so that $h^{0}\left(\mathcal{O}_{S}(-M)\right)=0$. This implies $h^{2}\left(\mathcal{O}_{S}(M)\right)=0$. Finally $h^{1}\left(\mathcal{O}_{S}(M)\right)=0$ follows from $\chi\left(\mathcal{O}_{S}(M)\right)=0$.

We recall that the Nikulin lattice is an even lattice of rank 8 generated by $n_{1} \ldots n_{8}$ and $m=\frac{1}{2}\left(n_{1}+\cdots+n_{8}\right)$, the product of which is uniquely defined by the condition $n_{i} n_{j}=-2 \delta_{i j}$, [M] 5.3. Notice also that the set of generators $\left\{n_{1}, \ldots, n_{8}, m\right\}$ is unique up to multiplying each element by -1 . Let $i=1 \ldots 8$, clearly $\mathcal{O}_{S}(M)$ and $\mathcal{O}_{S}\left(N_{i}\right)$ generate a Nikulin lattice in Pic $S$. Moreover this set of generators is uniquely defined by the condition 
$h^{0}\left(\mathcal{O}_{S}\left(N_{i}\right)\right)=1$. We will denote such a lattice by $\mathbb{L}_{S}$ and say that $\mathbb{L}_{S}$ is the Nikulin lattice of $(S, \mathcal{L}, \mathcal{M})$. The next property is well known

Theorem 2.2. For a general Nikulin surface of genus $g$ one has

$$
\text { Pic } S=\mathbb{Z} \mathcal{L} \oplus \mathbb{L}_{S} \text {. }
$$

It easily follows from the theorem and the preceeding remarks that the assignement $(S, \mathcal{L}, \mathcal{M}) \longrightarrow(S, \mathcal{L})$ induces a generically injective rational map from the moduli space of Nikulin surfaces of genus $g$ to $\mathcal{F}_{g}$. We will say that the image of this map is the Nikulin locus in $\mathcal{F}_{g}$ and denote it by

$$
\mathcal{F}_{g}^{N}
$$

Our aim is to show that $\mathcal{F}_{g}^{N}$ is rational for $g=8$. To this purpose let us summarize some geometry of projective models a Nikulin surface $(S, \mathcal{L}, \mathcal{M})$ of genus $g$, cfr. GS, SvG]. To begin we have the commutative diagram:

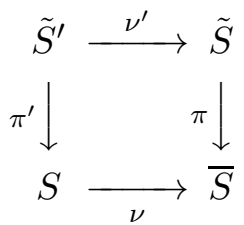

where $\pi^{\prime}$ is the double covering defined by $N$ and $\nu$ is the contraction of $N$. Let $E_{i}=\pi^{\prime-1}\left(N_{i}\right), i=1 \ldots 8$, then $E_{i}$ is an exceptional line on the smooth surface $\tilde{S}^{\prime}$. It turns out that $\nu^{\prime}$ is the contraction of $E_{1}+\cdots+E_{8}$ and that $\tilde{S}$ is a minimal K3 surface. Moreover $\pi$ is the quotient map of a symplectic involution $\iota: \tilde{S} \rightarrow \tilde{S}$ and its 8 fixed points are $\nu^{\prime}\left(E_{1}\right) \ldots \nu^{\prime}\left(E_{8}\right)$. Then it follows that $\pi$ is branched exactly on the even set of nodes

$$
o_{1}:=\nu\left(N_{1}\right), \ldots, o_{8}:=\nu\left(N_{8}\right)
$$

of $\bar{S}$, in particular Sing $\bar{S}=\left\{o_{1} \ldots o_{8}\right\}$. On $\tilde{S}$ we fix the polarization

$$
\tilde{\mathcal{L}}:=\pi^{*} \overline{\mathcal{L}}
$$

where $\overline{\mathcal{L}}$ is the line bundle $\nu_{*} \mathcal{L}$. Then $(\tilde{S}, \tilde{\mathcal{L}})$ is a K3 surface of genus $2 g-1$ and $\iota^{*} \tilde{\mathcal{L}} \cong \tilde{\mathcal{L}}$. The action of $\iota$ on $H^{0}(\tilde{\mathcal{L}})$ can be described as follows:

$$
H^{0}(\tilde{\mathcal{L}})=\pi^{*} H^{0}(\overline{\mathcal{L}}) \oplus H^{0}\left(\mathcal{I}_{f} \otimes \tilde{\mathcal{L}}\right)
$$

where $f=\pi^{-1}(\operatorname{Sing} \bar{S})$ is the set of the fixed points of $\iota$ and $\mathcal{I}_{f}$ is its ideal sheaf in $\tilde{S}$. The previous summands respectively are the +1 and -1 eigenspaces of $\iota$. We have $\operatorname{dim} H^{0}(\overline{\mathcal{L}})=g+1$ and $\operatorname{dim} H^{0}\left(\mathcal{I}_{f} \otimes \tilde{\mathcal{L}}\right)=g-1$.

Now let us consider the rational map $f_{\tilde{\mathcal{L}}}: \tilde{S} \rightarrow \mathbf{P}^{2 g-2}:=\mathbf{P} H^{0}(\tilde{\mathcal{L}})^{*}$ defined by $\tilde{\mathcal{L}}$ and the linear subspaces $\mathbf{P}^{g}:=\mathbf{P} H^{0}(\tilde{\mathcal{L}})^{*}$ and $\mathbf{P}^{g-2}:=\mathbf{P} H^{0}\left(\mathcal{I}_{f} \otimes \tilde{\mathcal{L}}\right)^{*}$ of $\mathbf{P}^{2 g-1}$ defined by the previous direct sum. Then we can add to the picture 
the following commutative diagram:

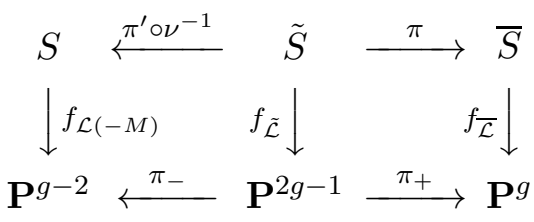

Here $\pi_{+}$and $\pi_{-}$respectively are the linear projections of centers $\mathbf{P}^{g-2}$ and $\mathbf{P}^{g}$. Notice that $\iota$ acts on $\mathbf{P}^{2 g-1}$ as a projective involution and that $\mathbf{P}^{g-2}$ and $\mathbf{P}^{g}$ are its projectivized eigenspaces. It is easy to see that the vertical maps are defined, from left to right, by the line bundles $\mathcal{L}(-M), \tilde{\mathcal{L}}, \overline{\mathcal{L}}$. We omit any further detail. From now on we fix the notations

$$
\mathcal{H}:=\mathcal{L}(-M), \mathcal{A}:=\mathcal{L}(-2 M) .
$$

We will use the following well known facts.

Proposition 2.3. For a general Nikulin surface of genus $g \geq 5$ the line bundle $\mathcal{H}$ is very ample.

Proof. See [GS] lemma 3.1

Proposition 2.4. For a general Nikulin surface of genus $g \geq 8$ a general member of $|\mathcal{A}|$ is a smooth irreducible curve of genus $g-8$.

Proof. We only sketch the standard proof: assume $g \geq 8$, then it follows from Riemann-Roch that $h^{0}(\mathcal{A}) \geq 1$. Let $A \in|\mathcal{A}|$, then $A+N$ belongs to $|\mathcal{L}|$ and we have the standard exact sequence

$$
0 \rightarrow \mathcal{A} \rightarrow \mathcal{L} \rightarrow \mathcal{O}_{N}(A) \rightarrow 0 .
$$

Moreover $\mathcal{O}_{N}(A)$ is the trivial sheaf $\mathcal{O}_{N}$ and it is easy to show that the restriction $H^{0}(\mathcal{L}) \rightarrow H^{0}\left(\mathcal{O}_{N}\right)$ is surjective. Passing to the associated long exact sequence it follows $h^{1}(\mathcal{A})=h^{1}(\mathcal{L})=0$. This implies that $|\mathcal{A}|$ is base point free and the statement for $g \geq 9$. For $g=8$ we have $A^{2}=-2$ so that $A$ is an isolated curve with $p_{a}(A)=0$. If $A$ is not integral one can deduce that Pic $S$ has rank $\geq 10$ : a contradiction for a general $S$.

Now, for a general Nikulin surface $S$ of genus $g \geq 5$, we consider the embedding

$$
S \subset \mathbf{P}^{g-2}
$$

defined by $f_{\mathcal{H}}$. Let $C \in|\mathcal{L}|$ : since $(C-M) N_{i}=1$ it follows that $N_{1} \ldots N_{8}$ are embedded as lines. Let $A \in|\mathcal{A}|, A$ is embedded as a curve of degree $2 g-10$. A general $A$ is integral of genus $g-8$, hence $h^{1}\left(\mathcal{O}_{A}(1)\right)=0$ for degree reasons. Since $\mathcal{H}(-A) \cong \mathcal{O}_{S}(M)$, we have the exact sequence

$$
0 \rightarrow \mathcal{O}_{S}(M) \rightarrow \mathcal{O}_{S}(1) \rightarrow \mathcal{O}_{A}(1) \rightarrow 0 \text {. }
$$

On the other hand we know that $h^{i}\left(\mathcal{O}_{S}(M)\right)=0, i=0,1,2$. Therefore, passing to the associated long exact sequence, we obtain

$$
0 \rightarrow H^{0}\left(\mathcal{O}_{S}(1)\right) \rightarrow H^{0}\left(\mathcal{O}_{A}(1)\right) \rightarrow 0 .
$$

This shows that 
Proposition 2.5. For a general Nikulin surface $S$ of genus $g \geq 8$ a general $A \in|\mathcal{A}|$ is embedded by $f_{\mathcal{H}}$ as a smooth irreducible curve spanning $\mathbf{P}^{g-2}$.

For a general Nikulin surface $S$ of genus $g \geq 8$ we also point out that

Proposition 2.6. In the projective model defined by $f_{\mathcal{H}}$ the lines $N_{1} \ldots N_{8}$ are bisecant lines to a smooth irreducible $A \in|\mathcal{A}|$.

Finally we can also consider the moduli space $\mathcal{D}_{g}^{\prime}$ of triples $(X, \mathcal{L}, \mathcal{H})$ such that $(X, \mathcal{L})$ is a $\mathrm{K} 3$ surface of genus $g$ and $\mathcal{H} \in \operatorname{Pic} S$ is a primitive big and nef element satisfying the following intersection properties:

$$
(\mathcal{H}, \mathcal{H})=2 g-6,(\mathcal{H}, \mathcal{L})=2 g-2 .
$$

As is well known this moduli space is an integral quasi projective variety and the assignement $(X, \mathcal{L}, \mathcal{H}) \longrightarrow(X, \mathcal{L})$ induces a generically injective morphism $\mathcal{D}_{g}^{\prime} \rightarrow \mathcal{F}_{g}$. Its image is an integral divisor we will denote as

$$
\mathcal{D}_{g}
$$

notice that $\operatorname{Pic} X=\mathbb{Z} \mathcal{L} \oplus \mathbb{Z} \mathcal{H}$ for a general triple $(X, \mathcal{L}, \mathcal{H})$, cfr. $[\mathrm{H}, \mathrm{BV}$.

It is clear that we have the inclusions

$$
\mathcal{F}_{g}^{N} \subset \mathcal{D}_{g} \subset \mathcal{F}_{g}
$$

This implies, by semicontinuity and the irreducibility of $\mathcal{D}_{g}^{\prime}$, that the above propositions 2.3, 2.4, 2.5 extend verbatim from the case of a general Nikulin surface to that of a general triple $(X, \mathcal{L}, \mathcal{H})$ and to its line bundles $\mathcal{H}$ and $\mathcal{A}:=\mathcal{L}(-2 M)$, where $\mathcal{O}_{S}(M):=\mathcal{L} \otimes \mathcal{H}^{-1}$. For such a general triple let

$$
X \subset \mathbf{P}^{g-2}
$$

be the embedding defined by $\mathcal{H}$. If $X$ is a Nikulin surface then $X$ contains 8 disjoint lines. This condition on $(X, \mathcal{L}, \mathcal{H})$ is not enough to have a Nikulin. For $g \geq 8$ we have $\mathcal{A} \mathcal{A}=2 g-18 \geq-2$, hence $|\mathcal{A}|$ is not empty. The next result characterizes the Nikulin locus $\mathcal{F}_{g}^{N}$ in $\mathcal{D}_{g}$, cfr. [GS] 3.2.

Theorem 2.7. Let $g \geq 8$ : a general triple $(X, \mathcal{L}, \mathcal{H})$ defines a point in $\mathcal{F}_{g}^{N}$ iff $X$ contains eight disjoint lines $N_{1} \ldots N_{8}$ which are bisecant to a curve $A \in|\mathcal{A}|$, that is, iff $A N_{1}=\cdots=A N_{8}=2$.

Proof. Assume $A N_{1}=\cdots=A N_{8}=2$ with $A \in|\mathcal{A}|$ and consider $A^{\prime}:=$ $2 H-A-N$, where $H \in|\mathcal{H}|$ and $N:=N_{1}+\cdots+N_{8}$. One computes $A^{2}=$ $A^{\prime 2}=A A^{\prime}=2 g-18$ and $H A=H A^{\prime}=2 g-10$. So we have $\left(A-A^{\prime}\right)^{2}=0$ and $H\left(A-A^{\prime}\right)=0$. This implies $A \sim A^{\prime}$ since $H$ is pseudoample. Then it follows $N \sim 2 H-2 A$ and $X$ is a Nikulin surface. The converse is immediate.

\section{Nikulin SURFACES OF GENUS 8 AND RATIONAL NORMAL SEXTiCs}

Let $S$ be a general Nikulin surface of genus 8 embedded in $\mathbf{P}^{6}$ by $|\mathcal{H}|$, then $|\mathcal{A}|$ contains a unique element $A$ which is embedded as a rational normal sextic. $S$ is in the irreducible component of its Hilbert scheme, the general 
point of which general point is a smooth $\mathrm{K} 3$ surface $\left(X, \mathcal{O}_{X}(1)\right)$ of genus 6 . Moreover one has

$$
\operatorname{Pic} X \cong \mathbb{Z} \mathcal{O}_{X}(1) \text {. }
$$

The Mukai-Brill-Noether theory for curves and K3 surfaces is definitely performed in genus 6 , cfr. [Mu, $\mathrm{Mu} 1$. It can be summarized as follows. A smooth hyperplane section $H$ of $X$ is a canonical curve of genus 6 :

Case (1) Assume that $H$ is not trigonal nor biregular to a plane quintic. Then $H$ is generated by quadrics, moreover there exists exactly one $H$-stable rank 2 vector bundle $\mathcal{E}$ on $X$ such that:

(i) $\operatorname{det} \mathcal{E} \cong \mathcal{O}_{Y}(1)$;

(ii) $h^{0}(\mathcal{E})=5$ and $h^{i}(\mathcal{E})=0$ for $i \geq 1$;

(iii) the determinant map det $: \wedge^{2} \bar{H}^{0}(\mathcal{E}) \rightarrow H^{0}\left(\mathcal{O}_{\mathbf{P}^{6}}(1)\right)$ is surjective.

Let $G(1,4) \subset \mathbf{P}^{9}:=\mathbf{P} \wedge^{2} H^{0}(\mathcal{R})^{*}$ be the Plücker embedding of the Grassmannian of 2-dimensional subspaces of $H^{0}(\mathcal{E})^{*}$. By (iii) the dual of det induces a linear embedding $\delta: \mathbf{P}^{6} \rightarrow \mathbf{P}^{9}$, moreover the construction yelds the commutative diagram

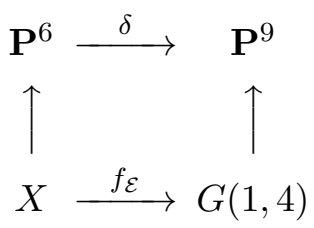

where the vertical maps are the inclusions and $f_{\mathcal{E}}$ is the embedding defined by $\mathcal{E}$. Fixing the identifications $\mathbf{P}^{6}:=\delta\left(\mathbf{P}^{6}\right)$ and $X:=f_{\mathcal{R}}(X)$ let us say in a simpler way that

$$
X \subset \mathbf{P}^{6} \cap G(1,4) \subset \mathbf{P}^{9} .
$$

Let

$$
T=\mathbf{P}^{6} \cdot G(1,4) \subset \mathbf{P}^{9},
$$

Mukai theory in genus 6 says also that:

(iv) $X$ is a quadratic section of $T$,

Since $X$ is a smooth quadratic section of $T$ it follows that $T$ is an integral 3-dimensional linear section of $G(1,4)$ with isolated singularities. Actually $T$ is a smooth Del Pezzo threefold of degree 5 if $X$ is sufficiently general.

Case (2) Assume that $H$ is either trigonal or biregular to a plane quintic. Then $H$ has Clifford index 1 and the following property holds true:

- there exists an integral curve $D \subset X$ such that either $D H=3$ and $D^{2}=0$ or $D H=5$ and $D^{2}=2$.

A general Nikulin surface of genus 8 occurs in case (1).

Proposition 3.1. Let $S \subset \mathbf{P}^{6}$ be a general Nikulin surface of genus 8 embedded by $f_{\mathcal{H}}$. Then $S$ is a quadratic section of a threefold $T$ as above. 
Proof. Pic $S$ is the orthogonal sum of rank $9 \mathbb{Z} \mathcal{L} \oplus \mathbb{L}_{S}$, where $\mathbb{L}_{S}$ is the Nikulin lattice generated by $\mathcal{O}_{S}(M), \mathcal{O}_{S}\left(N_{1}\right) \ldots \mathcal{O}_{S}\left(N_{8}\right)$. A standard computation we omit, shows that no divisor $D$ exists such that $D^{2}=0$ and $D H=3$ or $D^{2}=2$ and $D H=5$. This excludes case (2).

From now on we assume that $S$ is a general Nikulin surface of genus 8 , in particular we will assume that $S$ occurs in case (1) and that Pic $S \cong \mathbb{Z} \oplus \oplus \mathbb{L}_{S}$. We also assume that $S$ is embedded in $\mathbf{P}^{6}$ by $|\mathcal{H}|$. Then $S$ contains the rational normal sextic $A$ which is the unique element of $|\mathcal{A}|$. We want to study the restriction

$$
\mathcal{E}_{A}:=\mathcal{E} \otimes \mathcal{O}_{A}
$$

of the Mukai bundle $\mathcal{E}$ and discuss the possible cases. Of course we have $\mathcal{E}_{A}=\mathcal{O}_{\mathbf{P}^{1}}(m) \oplus \mathcal{O}_{\mathbf{P}^{1}}(n)$ with $m+n=6$.

Lemma 3.2. One has $m, n \geq 0$ so that $h^{0}\left(\mathcal{E}_{A}\right)=8$ and $h^{1}\left(\mathcal{E}_{A}\right)=0$.

Proof. Consider the commutative diagram

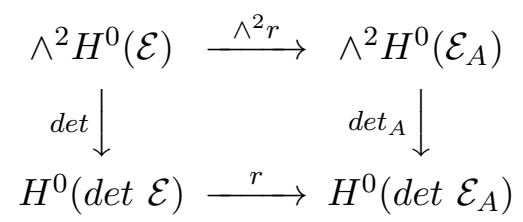

The restriction map $r$ is an isomorphism by 2.5 and det is surjective. This implies $m, n \geq 0$ : otherwise $\operatorname{det}_{A}$ would be the zero map.

Now we consider the surface $\mathbb{P}_{A}:=\mathbf{P} \mathcal{E}_{A}^{*}$ and its tautological map

$$
u_{A}: \mathbb{P}_{A} \rightarrow \mathbf{P}^{7}:=\mathbf{P} H^{0}\left(\mathcal{E}_{A}\right)^{*} .
$$

Since $m$ and $n$ are non negative, $u_{A}$ is a generically injective morphism with image a rational normal scroll of degree 6 . For it we fix the notation

$$
R:=u_{A}\left(\mathbb{P}_{A}\right) .
$$

We can assume $m \leq n$. Notice that $m$ is the minimal degree of a section of $\mathbb{P}_{A}$. If $m=0$ then $R$ is a cone over a rational normal sextic. If $m \geq 1$ then $u_{A}$ is an embedding. We consider the standard exact sequence

$$
0 \rightarrow \mathcal{E}(-A) \rightarrow \mathcal{E} \rightarrow \mathcal{E}_{A} \rightarrow 0 .
$$

Lemma 3.3. The associated long exact sequence is the following:

$$
0 \rightarrow H^{0}(\mathcal{E}) \rightarrow H^{0}\left(\mathcal{E}_{A}\right) \stackrel{\delta_{A}}{\rightarrow} H^{1}(\mathcal{E}(-A)) \rightarrow 0 .
$$

In particular one has $h^{0}(\mathcal{E})=5, h^{0}\left(\mathcal{E}_{A}\right)=8$ and $h^{1}(\mathcal{E}(-A))=3$.

Proof. Since $\mathcal{E}(-A)$ is $H$-stable and $H(H-2 A)<0$, it follows $h^{0}(\mathcal{E}(-A))=$ 0 . Furthermore we know that $h^{i}(\mathcal{E})=0$ for $i \geq 1$ and we have $h^{1}\left(\mathcal{E}_{A}\right)=0$ because $m, n \geq 0$. This implies the statement. 
Then the coboundary map $\partial_{A}: H^{0}\left(\mathcal{E}_{A}\right) \rightarrow H^{1}(\mathcal{E}(-A))$ defines a plane

$$
P_{A}:=\mathbf{P} \operatorname{Im} \partial_{A}^{*} \subset \mathbf{P}^{7} \text {. }
$$

Let $\mathbf{P}^{4}:=\mathbf{P} H^{0}(\mathcal{E})^{*}$. Then, dualizing the sequence and projectivizing the maps, we obtain the linear projection

$$
\alpha_{A}: \mathbf{P}^{7} \rightarrow \mathbf{P}^{4}:=\mathbf{P} H^{0}(\mathcal{E})^{*},
$$

of center $P_{A}$. Let $\mathbb{P}_{S}:=\mathbf{P} \mathcal{E}^{*}$, in turn $\alpha_{A}$ defines the commutative diagram

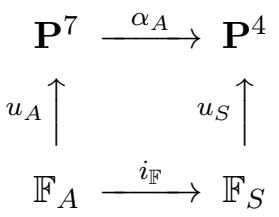

where $i_{\mathbb{F}}$ is the inclusion $\mathbb{F}_{A} \subset \mathbb{F}_{S}$ and the vertical arrows are the tautological maps. Furthermore let $G(2,8)$ be the Plücker embedding of the Grassmannian of 2-dimensional subspaces in $H^{0}\left(\mathcal{E}_{A}\right)^{*}$ and let $l \subset \mathbf{P}^{7}$ be a general line. Then the assignement $l \longrightarrow \alpha_{A}(l)$ defines a natural linear projection

$$
\lambda_{A}: G(2,8) \rightarrow G(1,4) .
$$

It follows immediately from the previous diagram that

Proposition 3.4. The next diagram is commutative:

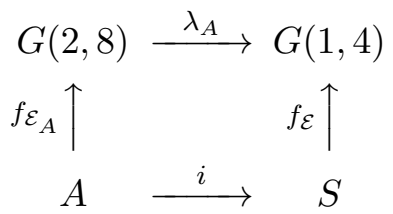

Here $i$ is the inclusion map and $f_{\mathcal{E}_{A}}$ and $f_{\mathcal{E}}$ are the maps associated to $\mathcal{E}_{A}$ and $\mathcal{E}$. We will profit of this construction in the next section, where the very special feature of the projection $\alpha_{A}$ will be described. For the moment we use the previous remarks to describe $\mathcal{E}_{A}$ for a general $S$.

Theorem 3.5. For a general Nikulin surface of genus 8 one has

$$
\mathcal{E}_{A}=\mathcal{O}_{\mathbf{P}^{1}}(3) \oplus \mathcal{O}_{\mathbf{P}^{1}}(3) \text {. }
$$

Proof. We have $\mathcal{E}_{A}=\mathcal{O}_{\mathbf{P}^{1}}(m) \oplus \mathcal{O}_{\mathbf{P}^{1}}(n)$ with $0 \leq m \leq n \leq 6$ and $m+n=6$. It suffices to show that $R$ is not a cone and that no rational section of degree 1 or 2 is contained in it. This indeed implies $m=3$. To this purpose consider the projected scroll $R^{\prime}=\alpha_{A}(R)$. Since $A$ is embedded in $G(1,4)$ as an integral sextic curve, the degree of $R^{\prime}$ is six. For any integral variety $Y \subset \mathbf{P}^{4}$ we denote by $\sigma_{Y}$ the variety in $G(1,4)$ parametrizing the lines intersecting $Y$. Let us exclude the cases $0 \leq m \leq 2$.

$m=0$. Then the scroll $R^{\prime}$ is a cone of vertex $o$ and $A$ is contained in $\sigma_{o}$. But $\sigma_{o}$ is a linear space of dimension four and $A$ would be a degenerate curve in it, which is excluded.

$m=1$. In this case $R^{\prime}$ contains a line $L$ intersecting every line of its ruling. Consider $\sigma_{L}$ : it is well known that $\sigma_{L}$ is a cone of vertex a point 
$l$ over the Segre embedding $\mathbf{P}^{1} \times \mathbf{P}^{2} \subset \mathbf{P}^{5}$. Since $A \subset \sigma_{L}$ it follows that $\sigma_{L} \subset \mathbf{P}^{6}=<A>$. Moreover $\mathbf{P}^{6}$ is the linear space tangent to $G(1,4)$ at the parameter point of $L$. But then $T=\sigma_{L}$ : a contradiction.

$m=2$. We can assume that $R^{\prime}$ contains a smooth conic $K$ intersecting all the lines of the ruling of $R^{\prime}$. Let $P$ be the supporting plane of $K$, then $S$ is contained in the codimension 1 Schubert cycle $\sigma_{P}$. This is endowed with a ruling of 4-dimensional smooth quadrics having the dual plane $P^{*}$ as the base locus. Every element of such a ruling is the Plücker embedding of the Grassmannian of the lines contained in a hyperplane through $P$. Notice also that Sing $\sigma_{P}=P^{*}$. Then, since $S$ is a smooth complete intersection of three hyperplane sections of $G(1,4)$ and of a quadric section, it follows that $S \cap P^{*}=\emptyset$. But then this ruling of quadrics of $\sigma_{P}$ cuts on $S$ a base pont free pencil $|D|$ such that $D^{2}=0$ and $D H=4$. This is excluded again by a standard computation in the Picard lattice of a general Nikulin surface.

\section{NikULIN SURFACES OF GENUS 8 AND SYMMETRIC CUBIC THREEFOLDS}

In what follows a symmetric cubic threefold is just a cubic hypersurface in $\mathbf{P}^{4}$ whose equation is the determinant of a symmetric $3 \times 3$ matrix of linear forms. As is well known the family of symmetric cubic threefolds is irreducible and its quotient under the action of $P G L(5)$ has finitely many orbits. One of them is open and it is the projective equivalence class of $\operatorname{Sec} B$, where $B$ is a rational normal quartic in $\mathbf{P}^{4}$. We will say that $\operatorname{Sec} B$ is the symmetric cubic threefold, Moreover we fix $B$ and the notation

$$
V:=\operatorname{Sec} B \text {. }
$$

The symmetric cubic threefold is nicely related to special embeddings $A \subset G(1,4)$ of a rational normal sextic and to the family of Nikulin surfaces of genus 8 . To see this we go back to the previous section, keeping the same notation. Since $S$ is a general Nikulin surface of genus 8 we will assume, by 3.5 , that $\mathbb{F}_{A}$ is the Hirzebruch surface $\mathbb{F}_{0}$ i.e. $\mathbb{F}_{A}=\mathbf{P}^{1} \times \mathbf{P}^{1}$. We consider again the linear projection

$$
\alpha_{A}: R \rightarrow \mathbf{P}^{4}
$$

which is uniquely defined by $S$. Fixing the sextic rational normal scroll $R \subset \mathbf{P}^{7}$, the family of the linear maps $\beta: R \rightarrow \mathbf{P}^{4}$ is parametrized by the Grassmannian $G(5,8)$. It follows from double points formula that:

Lemma 4.1. Let $\beta: R \rightarrow \mathbf{P}^{4}$ be a general linear projection. Then $\beta$ is a generically injective morphism and $\operatorname{Sing} \beta(R)$ is a set of six non normal double points with two branches.

Actually each point $o \in \operatorname{Sing} \beta(R)$ has embedding dimension 3, since $R$ is a scroll, and quadratic tangent cone of rank two. It is easy to see that this is not the case for $\alpha_{A}: R \rightarrow \mathbf{P}^{4}$ and that the projected surface $R^{\prime}=\alpha_{A}(R) \subset \mathbf{P}^{4}$ has an interesting feature. Indeed we have

$$
A \subset S \subset T=G(1,4) \cap \mathbf{P}^{6}
$$


where the Nikulin surface $S$ is general. Then $S$ contains the eight disjoint lines $N_{1} \ldots N_{8}$. Let $i=1 \ldots 8$, we observe that $N_{i}$ parametrizes a pencil of lines in $\mathbf{P}^{4}$. Moreover $A N_{i}=2$ so that $N_{i}$ is a bisecant line to $A$.

On the other hand $R^{\prime}$ is precisely the union of the lines parametrized by $A$. Let $o_{i}$ be the center of the pencil of lines parametrized by $N_{i}$ and let $Z_{i}=A \cdot N_{i}$. Since $S$ is general $Z_{i}$ is a 0 -dimensional scheme of length 2 in $S$, moreover the next lemma is immediate

Lemma 4.2. $\alpha_{A} / Z_{i}: Z_{i} \rightarrow \mathbf{P}^{4}$ contracts $Z_{i}$ to the point $o_{i}$.

The lemma implies that $\alpha_{A}$ contracts the scheme $Z=\cup Z_{i}$, of length 16 , to a scheme of length 8 supported on the points $o_{1} \ldots o_{8}$. By the previous double points formula this is impossible if $\alpha_{A}$ is not an embedding on at most finitely many points. Hence Sing $R^{\prime}$ contains a curve.

At first we observe that:

Lemma 4.3. Sing $R^{\prime}$ is an integral curve of degree $m$ with $3 \leq m \leq 4$.

Proof. By the proof of theorem $3.5 \alpha_{A}: R \rightarrow \mathbf{P}^{4}$ is a generically injective morphism, moreover $R^{\prime}$ is not a cone nor contains curves of degree $\leq 2$. On the other hand a general hyperplane section of $R^{\prime}$ is an integral sextic curve in $\mathbf{P}^{3}$, hence the number of its singular points is $\leq 4$.

It is clear that $\alpha_{A}: R \rightarrow R^{\prime}$ is the normalization map of $R^{\prime}$. Let $\tilde{B}$ be the pull-back of Sing $R^{\prime}$ by $\alpha_{E}$, then $\tilde{B}$ is a curve of type $(a, b)$ in $R=\mathbf{P}^{1} \times \mathbf{P}^{1}$ such that $a+3 b=2 m$. It is easy to compute the only possible cases:

(1) $m=4$ and $(a, b)=(2,2)$

(2) $m=4$ and $(a, b)=(5,1)$

(3) $m=3$ and $(a, b)=(3,1)$

Note that the type $(2,2)$ in case (1) implies that each line of $R^{\prime}$ is bisecant to Sing $R^{\prime}$. We do not discuss these cases. We only state as a claim the next theorem, which describes what happens in the case of our interest.

Theorem 4.4. For a general Nikulin surface $S$ as above we have:

- Sing $R^{\prime}$ is a rational normal quartic curve $B$ and $R^{\prime}$ is the complete intersection of $V=\operatorname{Sec} B$ and a quadric through $B$.

Moreover:

$\circ R$ is $\mathbf{P}^{1} \times \mathbf{P}^{1}$ and $\tilde{B}$ is a smooth curve of type $(2,2)$ in it,

- $T=\langle A\rangle \cdot G(1,4)$ is a smooth quintic Del Pezzo threefold.

In the next section an integral family $\mathbb{P}$ of Nikulin surfaces of genus 8 is constructed, whose general member satisfies the conditions stated above. Then $\mathbb{P}$ is used to prove the rationality of the moduli space $\mathcal{F}_{8}^{N}$.

A byproduct of the proof is that $\mathbb{P}$ dominates $\mathcal{F}_{8}^{N}$, hence the previous theorem follows. The construction of a surface in this family offers an explicit geometric construction of a general Nikulin surface in genus 8 . 


\section{Special Rational normal Sextics in the $G(1,4)$}

To construct the required family and to use it later, we construct at first a family of rational normal sextics $A \subset G(1,4)$ which are specially embedded. To begin we fix a rational normal quartic curve $B \subset \mathbf{P}^{4}$ and the symmetric cubic threefold $V:=\operatorname{Sec} V$. Let us also fix the notation

$$
\mathbf{P}^{2}:=\operatorname{Hilb}_{2}(B) \text {. }
$$

Any point $z \in \mathbf{P}^{2}$ is an effective divisor $b_{z} \in \operatorname{Div} B$ of degree two, the line

$$
V_{z}:=<b_{z}>
$$

is a bisecant line to $B$. We will denote its parameter point in $G(1,4)$ by $z$. Let $\mathbb{I} \subset \mathbf{P}^{4} \times G(1,4)$ be the universal line over $G(1,4)$, in it we have

$$
\tilde{V}:=\left\{(x, z) \in \mathbf{P}^{4} \times G(1,4) / x \in V_{z}\right\} .
$$

$\tilde{V}$ is endowed with its natural projection $v: \tilde{V} \rightarrow G(1,4)$, we set

$$
Z:=v(\tilde{V}) \text {. }
$$

Then we have $Z \subset G(1,4) \subset \mathbf{P}^{9}$, the next property is standard.

\section{Lemma 5.1.}

(1) $v: \tilde{V} \rightarrow Z$ is the projective bundle $\mathbf{P} T_{\mathbf{P}^{2}}^{*}$.

(2) $Z$ is the 3 -Veronese embedding of $\mathbf{P}^{2}$.

(3) $Z$ is embedded in $G(1,4)$ as a surface of cohomology class $(3,6)$.

Actually (2) and (3) follow from $c_{1}\left(T_{\mathbf{P}^{2}}\right) \cong \mathcal{O}_{\mathbf{P}^{2}}(3)$ and $c_{2}\left(T_{\mathbf{P}^{2}}\right)=3$.

On the other hand we consider the morphism $\sigma: \tilde{V} \rightarrow V$, induced on $\tilde{V}$ by the projection in $\mathbf{P}^{4}$. The structure of $\sigma$ is well known as well.

Lemma 5.2. $\sigma: \tilde{V} \rightarrow V$ is the contraction to $B$ of the divisor

$$
E:=\left\{(x, z) \in V \times Z / x \in b_{z}\right\} .
$$

Moreover $E$ in biregular to $B \times B$.

Proof. Let $x \in V-B$. Since $B$ is not degenerate of degree four, there exists a unique bisecant line $V_{z}$ passing through $x$. Hence $\sigma$ is birational and $\sigma^{-1}(x)=(x, z)$. Obviously $\sigma$ contracts $E$. Finally let $f: B \times B \rightarrow E$ be the morphism which is so defined: $f\left(x_{1}, x_{2}\right)=\left(x_{1}, z\right)$, where $b_{z}:=x_{1}+x_{2}$. For every $z \in Z$ one has $B \cdot V_{z}=b_{z}$. This implies that $f$ is biregular.

Now we consider on $\tilde{V}$ the linear systems

$$
|H|:=\left|\sigma^{*} \mathcal{O}_{\mathbf{P}^{4}}(1)\right| \text { and }|F|:=\left|v^{*} \mathcal{O}_{\mathbf{P}^{2}}(1)\right| .
$$

We have Pic $\tilde{V} \cong \mathbb{Z}[H] \oplus \mathbb{Z}[F]$ and we want to study with some detail

$$
|2 H-E| \text {. }
$$

At first we observe that $(2 H-E) F^{2}=0$. Indeed every fibre $V_{z} \times\{z\}$ of $v$ has numerical class $F^{2}$ in the Chow $\operatorname{ring} C H^{*}(\tilde{V})$. Since $B \cdot V_{z}=b_{z}$ we have $E F^{2}=2$ and hence $(2 H-E) F^{2}=0$. On the other hand we have 
$(2 H-E) H^{2}=2 H^{3}=6$. Hence it follows $2 H-E \sim 2 F$. Let $\mathcal{I}_{B}$ be the ideal sheaf of $B$ in $\mathbf{P}^{4}$, the next lemma then easily follows.

Lemma 5.3. The linear system $|2 F|$ is the strict trasform of the linear system $\left|\mathcal{I}_{B}(2)\right|$ by the birational morphism $\sigma: \tilde{V} \rightarrow V$.

It is clear from the lemma that to give $R \in|2 F|$ is equivalent to give the pull-back by $v$ of a conic, embedded by the 3 -Veronese map of $\mathbf{P}^{2}$ as a sextic curve in $Z \subset G(1,4)$.

Definition 5.1. $\mathbb{A}=\left|\mathcal{O}_{\mathbf{P}^{2}}(2)\right|$ is the family of embedded sextic curves

$$
A \subset Z \subset G(1,4) \text {. }
$$

We will say that $A \in \mathbb{A}$ is a special rational normal sextic of $G(1,4)$.

In particular, the linear space $\langle A\rangle$ is a $\mathbf{P}^{6}$ for each $A \in \mathbb{A}$. For a given $A \in \mathbb{A}$ we will keep the following notations:

$$
R=v^{*} A, R^{\prime}=\sigma_{*} R, T:=<A>\cdot G(1,4) .
$$

Now assume $A$ is general. We remark that the inclusion of $\mathbf{P}^{1}$-bundles $R \subset \tilde{V}$ is induced by the exact sequence

$$
0 \rightarrow \mathcal{E}_{Z}(-A) \rightarrow \mathcal{E}_{Z} \rightarrow \mathcal{E}_{A} \rightarrow 0,
$$

where $\mathcal{E}_{Z}=T_{\mathbf{P}^{2}}$ is the restriction of the universal bundle of $G(1,4)$ and hence $\mathcal{E}_{A}=T_{\mathbf{P}^{2}} \otimes \mathcal{O}_{A}$. Due to our generality assumption on $A$, the splitting of $\mathcal{E}_{A}$ is balanced. This implies the next lemma.

Lemma 5.4. $\mathcal{E}_{A}=\mathcal{O}_{\mathbf{P}^{1}}(3) \oplus \mathcal{O}_{\mathbf{P}^{1}}(3)$.

Next we want to study the morphism $v / E: E \rightarrow \mathbf{P}^{2}$. Since $\mathbf{P}^{2}$ is $\operatorname{Hilb}_{2}(B)$ a conic in it is distinguished, namely the diagonal

$$
D:=\left\{z \in \mathbf{P}^{2} / b_{z}=2 x\right\} .
$$

The double covering of $\mathbf{P}^{2}$ branched in $D$ can be viewed as follows. Let

$$
P:=\left\{(p, t) \in \mathbf{P}^{2} \times \mathbf{P}^{2 *} / p \in t \text { and } t \text { is tangent to } D\right\} .
$$

Then the projection $P \rightarrow \mathbf{P}^{2}$ is the $2: 1$ cover branched on $D$. In particular its fibre at $p$ is the set $\left\{t^{\prime}, t^{\prime \prime}\right\}$ of the tangent lines to $D$ passing through $p$.

Lemma 5.5. $v / E: E \rightarrow \mathbf{P}^{2}$ is the double covering branched on $D$.

Proof. Let $x \in B=\operatorname{Sing} V$ then $\sigma^{*}(x)$ is $\{x\} \times B$. Moreover $v\left(\sigma^{*} x\right)$ is the line $t_{x}:=\{x+y, y \in B\} \subset \mathbf{P}^{2}$. As is well known $t_{x}$ is tangent to $D$ at $2 x$. Let $z \in \mathbf{P}^{2}$ and let $b_{z}=x+y$, we just remark that the fibre of $v / E$ at $z$ is naturally bijective to $\left\{t_{x}, t_{y}\right\}$. This implies the statement.

Proposition 5.6. Let $A \in \mathbb{A}$ be general and let $\tilde{B}:=R \cdot E$, then $R$ is $\mathbf{P}^{1} \times \mathbf{P}^{1}$ and $\tilde{B}$ is a smooth curve in it of type $(2,2)$.

Proof. By lemma 5.4 we have $R=\mathbf{P}\left(\mathcal{E}_{A}\right)=\mathbf{P}^{1} \times \mathbf{P}^{1}$. Since $|R|$ is base point free, the rest of the statement is true for every $R$ transversal to $E$. 
For any $A \in \mathbb{A}$ we now consider the schematic image $R^{\prime}:=\sigma_{*}(R)$ of $R$. By lemma 5.3 $R^{\prime}$ is a quadro cubic complete intersection. More precisely we have $R^{\prime}=V \cdot Q$, where $Q$ is a quadric through $B$. The ruling of lines of $R^{\prime}$ is parametrized by $A$. In order to prove the next proposition the next lemma is useful. The proof is an exercise on the geometry of $G(1,4)$ we omit.

Lemma 5.7. A three dimensional linear section of $G(1,4)$ is not a smooth threefold iff it is contained in a codimension one Schubert cycle.

Proposition 5.8. Let $A \in \mathbb{A}$ be general and let $T=<A>\cdot G(1,4)$. Then $T$ is a smooth quintic Del Pezzo threefold.

Proof. Since $A$ is general we can assume that $\mathcal{E}_{A}=\mathcal{O}_{\mathbf{P}^{1}}(3) \oplus \mathcal{O}_{\mathbf{P}^{1}}(3)$ and that $R^{\prime}=V \cap Q$, with $Q$ a smooth quadric. Assume that $T$ is singular, then $T$ is contained in a Schubert cycle of codimension one, that is, in the Chow variety of a plane $P \subset \mathbf{P}^{4}$. Now $V$ does not contain any plane. To see this observe that $V$ is a hyperplane section of the secant variety $S e c Y$ of tte Veronese variety $Y$ in $\mathbf{P}^{5}$. Then any hyperplane section through a plane is the secant variety $V^{\prime}$ of the degenerate quartic $B^{\prime} \cup B^{\prime \prime}$, where $B^{\prime}, B^{\prime \prime}$ are smooth conics and $B^{\prime} \cap B^{\prime \prime}$ is one point. On the other hand $Q$ does not contain any plane as well, since it is smooth. Hence $P \cap Q \cap V$ is a curve of degree $m \leq 2$ contained in $R^{\prime}$. It is easy to see that its pull back by $\sigma / R: R \rightarrow R^{\prime}$ is a section of $R$ having self intersection $\geq-2$. This is impossible if $\mathcal{E}_{A}$ is balanced as above. Hence $T$ is smooth.

For a general $A \in \mathbb{A}$ we know that: $R$ is $\mathbf{P}^{1} \times \mathbf{P}^{1}$ and $\sigma / R: R \rightarrow R^{\prime}$ is the normalization map. The curve $\tilde{B}=(\sigma / R)^{*} \operatorname{Sing} R^{\prime}$ is smooth of type $(2,2)$. $T$ is smooth. We can summarize the situation as follows.

Theorem 5.9. For a general $A \in \mathbb{A}$ the statement of theorem 4.4 holds.

\section{Geometry of Nikulin surfaces of genus 8}

We have not yet used our family $\mathbb{A}$, of special embeddings of $\mathbf{P}^{1}$ in $G(1,4)$, to construct Nikulin surfaces of genus 8 , nor we have considered the special feature of these embeddings. About this we can say in short that a general $A \in \mathbb{A}$ admits a one dimensional family of bisecant lines which are contained in $T$. Moreover the union of them is a quadratic section of $T$. To see this quickly we fix a general $A \in \mathbb{A}$ and consider the complete intersection

$$
R^{\prime}=Q \cap V \text {. }
$$

Since $A$ is general we can assume that $Q \in\left|\mathcal{I}_{B}(2)\right|$ is general, then it is known that $Q$ is a smooth quadric. We recall from [FV, FV1] that the tangential quadratic complex of $Q$ is just the family

$$
W \subset G(1,4)
$$

parametrizing the lines which are tangent to $Q$. As is well known $W$ is a quadratic complex. In other words it is a quadratic section of $G(1,4)$. 
Actually $W$ is singular and has two orbits under the action of PGL(5): $W-\operatorname{Sing} W$ and Sing $W$, where the multiplicity is two. Finally:

$$
\text { Sing } W=F(Q),
$$

where $F(Q)$ denotes the Hilbert scheme of lines in $Q . F(Q)$ is embedded in $G(1,4)$ as the image of $\mathbf{P}^{3}$ under the 2-Veronese map.

Lemma 6.1. $W$ does not contain $T$.

Proof. Let $r, r^{\prime}$ be disjoint lines of the ruling of $R^{\prime}$ and $L:=<r \cup r^{\prime}>$. We consider the Grassmannian $G_{L} \subset G(1,4)$ of lines of $L$, which is embedded by its Plücker map. Since $r \cap r^{\prime}$ is empty, it easily follows that $q_{L}:=T \cap G_{L}$ is a conic containing the parameter points of $r$ and $r^{\prime}$. Its corresponding quadric $Q_{L}^{\prime} \subset L$ is not in $Q$. Otherwise we would have $q_{L} \subset \operatorname{Sing} W$, which is impossible because $\langle A\rangle \cdot \operatorname{Sing} W=A$. This indeed follows because $A$ is the image of a skew cubic of $\mathbf{P}^{3}$ : since its ideal in $\mathbf{P}^{3}$ is generated by three independent quadrics, then $A$ is cut on $\operatorname{Sing} W$ by the codimension three linear space $\langle A\rangle$. Since $r \cap r^{\prime}=\emptyset$, it is also true that $Q_{L}^{\prime}$ is either smooth or union of two disjoint planes. Let $Q_{L}=Q \cap L$, then $Q_{L}$ is smooth and the intersection $Q_{L} \cdot Q_{L}^{\prime}$ contains the skew lines $r$ and $r^{\prime}$. Let $r^{\prime \prime} \in\left|\mathcal{O}_{Q_{L}^{\prime}}(r)\right|$ be general. After the preceding remarks it is very easy to deduce that $r^{\prime \prime}$ is not tangent to $Q$. Hence $q_{L}$ is not in $W$ and $T$ is not in $W$.

$W$ cuts on $T$ a surface which defines an element of the Hilbert scheme of K3 surfaces of genus 6 in $G(1,4)$. We will see in a moment that this surface is a scroll in $T$ singular along $A$. To describe this scroll we consider the ruled surface over $B$ constructed as follows.

For each $o \in B$ consider in $\mathbf{P}^{2}$ the divisor of degree two of $A$

$$
n_{0}:=v_{*}\left(R \cdot \sigma^{*}(o)\right) \text {. }
$$

Then $n_{o}$ defines the plane $P_{o}:=<\sigma_{*} v^{*} n_{o}>$ and the pencil of lines of $P_{o}$ of center $o$. We denote this pencil by $N_{o}$. We also observe that $n_{o}$ is supported on two points for a general $o$ and that $\sigma_{*} v^{*} n_{o}$ is the union of the two lines in $R^{\prime}$ passing through $o$. Finally we define the ruled surface

$$
S_{A}:=\left\{(o, n) \in A \times T / n \in N_{o}\right\}
$$

and, via the projection $\tau: B \times T \rightarrow T$, its schematic image

$$
S_{A}^{\prime}:=\tau_{*}\left(S_{A}\right) \text {. }
$$

Definition 6.1. We say that $S_{A}^{\prime}$ is the fake K3 surface of $A$.

A standard computation in the Chow ring of $G(1,4)$ shows that $S_{A}^{\prime}$ has class $(4,6)$ in $C H^{4}(G(1,4))$. This implies that $S_{A}^{\prime}$ is embedded in $T$ as a surface of degree ten. On the other hand, since $T$ is a smooth quintic Del Pezzo threefold, Pic $T$ is generated by $\mathcal{O}_{T}(1)$. Therefore we conclude that

$$
S_{A}^{\prime} \in\left|\mathcal{O}_{T}(2)\right| \text {. }
$$

The next propositions say more. 
Proposition 6.2. $S_{A}^{\prime}$ is cut on $T$ by the tangential quadratic complex $W$.

Proof. In view of the latter remarks, it suffices to show that $W$ contains $S_{A}^{\prime}$. Let $(o, n) \in S_{A}$, then $n$ is a line of the pencil $N_{o}$. This is a pencil of the plane $P_{o}$ and it is generated by two lines, say $n^{\prime}, n^{\prime \prime}$, of the scroll $R^{\prime}$. Since $R^{\prime}=Q \cdot V$ and $Q$ is smooth, it follows that $P_{o} \cdot Q=n^{\prime} \cup n^{\prime \prime}$. Hence $P_{o}$ is a tangent plane to $Q$ at $o$ and each line of the pencil $N_{o}$ of center $o$ is tangent to $Q$. This implies $N_{o} \subset S_{A}$ and hence $S_{A}^{\prime} \subset W$.

Proposition 6.3. Sing $S_{A}^{\prime}=A$ and $A$ has multiplicity two.

Proof. Let us consider again the morphism $\tau: S_{A} \rightarrow S_{A}^{\prime}$ and a point $n \in$ $S_{A}^{\prime}$. From the definition of $S_{A}$ it follows that $\tau^{*} n$ is the scheme theoretic intersection of $B$ and the line parametrized by $n$. This easily implies that $\tau$ is birational and, moreover, that $n$ is singular iff parametrizes a bisecant line to $B$ contained in $R^{\prime}$, that is, $n \in A$ and $n$ has multiplicity two.

Remark 6.1. We only mention, without proofs, how the scroll $S_{A}$ is related to the Fano variety $F(T)$ of lines of the smooth threefold $T$. It is well known that every three dimensional linear section of $G(1,4)$ is projectively equivalent to $T$ and that $F(T)$ is a surface. The family of lines of $T$ has been studied in detail, cfr. [FN, TZ]. Actually $F(T)$ is $\mathbf{P}^{2}$, let $u: \mathbb{U} \rightarrow \mathbf{P}^{2}$ be the universal line and $\phi: \mathbb{U} \rightarrow G(1,4)$ the natural projection. For a plane curve $D \subset \mathbf{P}^{2}$ of degree $d$ we have that $\phi_{*} u^{*} D$ belongs to $\left|\mathcal{O}_{T}(d)\right|$. In particular we have $S_{A}=\phi_{*} u^{*} A^{\prime}$, where $A^{\prime}$ is a conic.

Finally we can pass to the construction of the predicted family of Nikulin surfaces of genus 8 .

We start with a general $A \in \mathbb{A}$. Then $A$ is a rational normal sextic in the smooth threefold $T$. Both $T$ and $A$ are projectively normal and generated by quadrics. Let $\mathcal{I}_{A}$ be the ideal sheaf of $A$ in $T$, we have $\operatorname{dim}\left|\mathcal{I}_{A}(2)\right|=9$.

Lemma 6.4. A general $S \in\left|\mathcal{I}_{A}(2)\right|$ is a smooth K3 surface of genus 6 .

Proof. Let $n \in A$ and $I_{n}=\left\{S \in\left|\mathcal{I}_{A}(2)\right| / n \in \operatorname{Sing} S\right\}$. Since $A$ is a smooth curve generated by quadrics, the codimension of $I_{n}$ is two. Hence $\cup_{n \in A} I_{n}$ is a proper closed set and a general $S \in\left|\mathcal{I}_{A}(2)\right|$ is smooth along $A$. On the other hand a general $S$ is smooth on $T-A$ by Bertini theorem.

We consider the morphism $\tau: S_{A} \rightarrow S_{A}^{\prime}$ and the linear projection

$$
\tau^{*}:\left|\mathcal{I}_{A}(2)\right| \rightarrow\left|\tau^{*} \mathcal{O}_{S_{A}}(2)\right| \text {. }
$$

Since $S_{A}^{\prime} \in\left|\mathcal{I}_{A}(2)\right|$ the image of $\tau^{*}$ is a linear system $\mathbb{I}$ of dimension 8. Since $A$ and $T$ are generated by quadrics, the fixed component of $\mathbb{I}$ is $\tau^{*} A$.

Lemma 6.5. Let $\pi: S_{A} \rightarrow B$ be the natural projection then

$$
\mathbb{I}=\tau^{*} A+\pi^{*}\left|\mathcal{O}_{\mathbf{P}^{1}}(8)\right| .
$$


Proof. Let $Q$ be a general quadric through $A$, not containing $S_{A}^{\prime}$. Then $Q \cdot S_{A}^{\prime}$ is a curve of degree 20 such that $A$ counts with multiplicity two. The residual component has degree 8. On the other hand let $o \in B$ and $N_{o}=\pi^{*}(o)$, then $N_{o}$ is embedded as a bisecant line to $o$. Indeed $N_{o}$ is the pencil of lines generated by the two lines of $R^{\prime}$ passing through $o$ and they define the two points of $N_{o} \cap A$. It is easy to deduce that the residual component is union of lines of the ruling of $S_{A}^{\prime}$. Hence we have $\tau^{*} Q=\tau^{*} A+M$, where $M \in\left|\mathcal{O}_{\mathbf{P}^{1}}(8)\right|$. The latter space has the same dimension of $\mathbb{I}$. Hence the statement follows.

Let $S \in\left|\mathcal{I}_{A}(2)\right|$ then $S$ is a smooth K3 surface. The lemma implies that $S$ contains eight disjoint lines $N_{1}, \ldots, N_{8}$ so that $A N_{i}=2, i=1 \ldots 8$, and

$$
S \cdot S_{A}^{\prime}=2 A+N_{1}+\cdots+N_{8} .
$$

We can definitely conclude, by theorem 2.7 , that $S$ is a Nikulin surface.

Theorem 6.6. Let $A \in \mathbb{A}$ be general, then a general $S \in\left|\mathcal{I}_{A}(2)\right|$ is a Nikulin surface of genus 8 .

\section{The Rationality of $\mathcal{F}_{8}^{N}$}

In this section we show the rationality of the moduli space $\mathcal{F}_{8}^{N}$ of genus 8 Nikulin surfaces and deduce theorem 4.4 from the method of proof.

To this purpose let us consider in $\mathbb{A} \times G(1,4)$ the universal families $\mathcal{R}$ and $\mathcal{T}$, respectively parametrizing pairs $(A, z)$ such that $z \in A$ and $z \in T, T:=$ $\langle A\rangle \cdot G(1,4)$. With some abuse we still denote by $\mathbb{A}$ a suitable open set of $\mathbb{A}$, parametrizing general and smooth rational normal sextics. Then we consider the ideal sheaf $\mathcal{I}$ of $\mathcal{R}$ in $\mathcal{T}$ and the vector bundle

$$
\mathcal{V}:=\alpha_{*}\left(\mathcal{I} \otimes \beta^{*} \mathcal{O}_{G(1,4)}(2)\right) \text {. }
$$

Here $\alpha$ and $\beta$ are the projections of $\mathbb{A} \times G(1,4)$ respectively onto $\mathbb{A}$ and $G(1,4)$. The construction defines the $\mathbf{P}^{9}$-bundle $\alpha: \mathbb{P} \rightarrow \mathbb{A}$, where we set

$$
\mathbb{P}:=\mathbf{P} \mathcal{V}
$$

The fibre $\mathbb{P}_{A}$ of $\alpha$ at $A \in \mathbb{A}$ is precisely the linear system of Nikulin surfaces $\left|\mathcal{I}_{A}(2)\right|$ considered in theorem 6.6. On the other hand we know that $\mathbb{A}=$ $\left|\mathcal{O}_{\mathbf{P}^{2}}(2)\right|$ and this is isomorphic to $\left|\mathcal{I}_{B}(2)\right|$ via the linear map

$$
\sigma_{*} \circ v^{*}: \mathbb{A} \rightarrow\left|\mathcal{I}_{B}(2)\right| \text {. }
$$

A general point of $\mathbb{P}$ is just a pair $(S, A)$ such that $S$ is a Nikulin surface of the linear system $\mathbb{P}_{A}$. More precisely its genus 8 polarization is uniquely defined from the pair as $\mathcal{L}=\mathcal{H}(A)$, where $\mathcal{H}=\mathcal{O}_{S}(1)$. Moreover we have $\mathcal{H}(-A) \cong \mathcal{O}_{S}(M)$ and $2 M \sim N_{1}+\cdots+N_{8}$, where the summands are the eight lines contained in $S \cap S_{A}$. We want to study the moduli map

$$
m: \mathbb{P} \rightarrow \mathcal{F}_{8}^{N}
$$

in order to prove that it is dominant. Then we consider two general elements $\left(S_{1}, A_{1}\right)$ and $\left(S_{2}, A_{2}\right)$ of $\mathbb{P}$. Let $i=1,2$ : as above the pair $\left(S_{i}, A_{i}\right)$ uniquely 
defines the triple of line bundles $\left(\mathcal{L}_{i}, \mathcal{H}_{i}, \mathcal{A}_{i}\right)$ where $\mathcal{A}_{i}=\mathcal{O}_{S_{i}}\left(A_{i}\right)$. Assume that the two elements have the same image by $m$. This is equivalent to say that there exists an isomorphism $f: S_{1} \rightarrow S_{2}$ such that $f^{*} \mathcal{H}_{2} \cong \mathcal{H}_{1}$ and $f^{*} \mathcal{A}_{2} \cong \mathcal{A}_{1}$. Now recall that $\mathcal{H}_{i}$ uniquely defines the Mukai vector bundle $\mathcal{E}_{i}$ of $S_{i}$ and that this is the restriction to $S_{i}$ of the universal bundle on $G(1,4)$. Hence it follows that $f^{*} \mathcal{E}_{2} \cong \mathcal{E}_{1}$ and that $f$ defines an isomorphism

$$
f^{*}: H^{0}\left(\mathcal{E}_{2}\right) \rightarrow H^{0}\left(\mathcal{E}_{1}\right) \text {. }
$$

This isomorphism and the property $f^{*} \mathcal{A}_{1} \cong \mathcal{A}_{1}$ imply the next lemma.

Lemma 7.1. $\left(S_{1}, A_{1}\right)$ and $\left(S_{2}, A_{2}\right)$ have the same image by $m$ iff there exists $a \in$ Aut $G(1,4)$ such that $a\left(S_{1}\right)=S_{2}$ and $a\left(A_{1}\right)=A_{2}$.

Due to the lemma we now study the group $G \subset$ Aut $G(1,4)$ leaving the set of pairs $\mathbb{P}$ invariant. We have Aut $G(1,4)=P G L(5)$ : let $a \in G$ then $a$ is induced by an automorphism of $\mathbf{P}^{4}$.

By definition $a$ leaves invariant the family of curves $\mathbb{A}$, therefore $G$ acts on $\mathbb{A}$ as well. In particular a leaves invariant the set of all lines in $\mathbf{P}^{4}$ parametrized by $\cup A, A \in \mathbb{A}$. This is precisely the family of bisecant lines to the rational normal quartic $B$, hence $a$ leaves $B$ invariant. Since $a$ is the identity iff $a / B: B \rightarrow B$ is the identity, we can conclude as follows.

Lemma 7.2. $G=$ Aut $B=\operatorname{PGL}(2)$.

Note that $\mathbb{A}$ is $\mathbf{P}^{5}$ and that $\operatorname{dim} \operatorname{PGL}(2)=3$. Therefore the quotient of the action of $G$ on the base of the projection map $\alpha: \mathbb{P} \rightarrow \mathbb{A}$ is rational. Indeed this quotient $\mathbb{A} / G$ is a surface. Therefore, since it is unirational, $\mathbb{A} / G$ is rational.

It is useful to reconsider the action of $G$ on $\mathbb{A}$ as follows. Recall that we have $\mathbb{A}=\left|\mathcal{O}_{\mathbf{P}^{2}}(2)\right|$, where $\mathbf{P}^{2}=\operatorname{Hilb}_{2}(B)$. What is the action of $G$ on $\left|\mathcal{O}_{\mathbf{P}^{2}}(2)\right|$ ? To answer we recall that $\operatorname{Hilb}_{2}(B)$ contains a distinguished conic, namely the diagonal $D$ already considered in section 5 .

$D$ parametrizes the family of tangent lines to $B$ and this family is left invariant by $G$. Hence $a \in G$ acts on $\left|\mathcal{O}_{\mathbf{P}^{2}}(2)\right|$ as an element $a^{\prime} \in$ Aut $\mathbf{P}^{2}$ leaving $D$ fixed. The map sending $a$ to $a^{\prime}$ is an isomorphism of $G$ and Aut $D$.

Lemma 7.3. The action of $G$ on $\mathbb{A}$ is generically faithful.

Proof. Let $G_{A} \subset G$ be the stabilizer of $A$ and $a \in G_{A}$. Consider $A$ as a general conic in $\mathbf{P}^{2}$. Then $a$ acts as an automorphism of $\mathbf{P}^{2}$ such that $a(A)=A$ and $a(D)=D$. Hence it follows $a(A \cap D)=A \cap D$. But, since $A$ is general, the set of four points $A \cap D$ is general. Hence $a$ is the identity.

Let $\overline{\mathbb{P}}:=\mathbb{P} / G$ and $\overline{\mathbb{A}}:=\mathbb{A} / G$, it follows from the previous lemmas that $\alpha: \mathbb{P} \rightarrow \mathbb{A}$ descends, over a non empty open set of $\overline{\mathbb{A}}$, to a $\mathbb{P}^{9}$-bundle

$$
\bar{\alpha}: \overline{\mathbb{P}} \rightarrow \overline{\mathbb{A}} \text {. }
$$

Since $\overline{\mathbb{A}}$ is rational, then $\overline{\mathbb{P}}$ is rational. Now the moduli map $m: \mathbb{P} \rightarrow \mathcal{F}_{8}^{N}$ descends to a rational map

$$
\bar{m}: \overline{\mathbb{P}} \rightarrow \mathcal{F}_{8}^{N}
$$


Moreover lemma 7.1 implies that $\bar{m}$ is generically injective. Since $\overline{\mathbb{P}}$ and $\mathcal{F}_{8}^{N}$ are integral of the same dimension, it follows that $\bar{m}$ is birational. This completes the proof of the next theorem.

Theorem 7.4. The moduli space of genus 8 Nikulin surfaces is rational.

\section{REFERENCES}

[BV] W. Barth and A. Verra Torsion on K3-sections, Symposia Math. 32 (1991) 1-24

[D] I. Dolgachev Classical Algebraic Geometry, A Modern View, Cambridge University Press, Cambridge UK (2012) pp. xii +639

[FN] M. Furushima and N. Nakayama The family of lines on the Fano threefold $V_{5}$, Nagoya Math. J. 116 (1989), 111122.

[FV] G. Farkas and A. Verra Moduli of theta characteristics via Nikulin surfaces, Math. Ann. 354 (2012) 465-496

[FV1] G. Farkas and A. Verra Prym varieties and moduli of polarized Nikulin surfaces, (2013) ArXiv 1503.05967 (2015)

[GH] V. Gritsenko and K. Hulek Moduli of polarized Enriques surfaces, to appear in K3 Surfaces and Their Moduli, Progress in Math, Birkhäuser. ArXiv 1502.02723 (2015)

[GS] A. Garbagnati and A. Sarti Projective models of K3 surfaces with an even set, Adv. Geom. 8 (2008) 413-440

[H] D. Huybrechts, Lectures on Algebraic K3 surfaces, http://www.math.uni-bonn.de/people/huybrech/K3Global.pdf (2014)

[M] D. Morrison, On K3 surfaces with large Picard number, Invent. Math. 75 (1984) $105-121$

[Mu] S. Mukai, An Introduction to Invariants and Moduli, Cambridge studies in adv. math. 81, Cambridge University Press, Cambridge (2012) 1-524

[Mu1] S.Mukai, Curves and Grassmannians, in Algebraic Geometry and related Topics, International Press, Cambridge MA (1993) 19-40

[N] V. Nikulin Finite automorphism groups of Kähler K3 surfaces, Trans. Moscow Math. Soc. 38 (1980) 71135.

[SvG] A. Sarti and B. van Geemen, Nikulin involutions on K3 surfaces, Math. Z. 255 (2007) 731-753

[TZ] M. Takagi and F. Zucconi, Geometries of lines and conics on the quintic Sel Pezzo 3-fold and its applications to varieties of power sums, Michigan Math. J. 61 (2012) 19-62

Dipartimento di Matematica e Fisica, Universitá Roma Tre, Largo San Leonardo Murialdo 100146 Roma, Italy

E-mail address: sandro.verra@gmail.com 